\title{
Algorithms of maximum likelihood data clustering with applications
}

\author{
Lorenzo Giada* and Matteo Marsili ${ }^{+}$ \\ * Max Planck Institut für Kolloid- und Grenzflächenforschung, 14424 Potsdam, Germany \\ ${ }^{+}$Istituto Nazionale per la Fisica della Materia (INFM), Trieste-SISSA Unit, \\ V. Beirut 2-4, Trieste I-34014
}

February 25, 2019

\begin{abstract}
We address the problem of data clustering by introducing an unsupervised, parameter free approach based on maximum likelihood principle. Starting from the observation that data sets belonging to the same cluster share a common information, we construct an expression for the likelihood of any possible cluster structure. The likelihood in turn depends only on the Pearson's coefficient of the data. We discuss clustering algorithms that provide a fast and reliable approximation to maximum likelihood configurations. Compared to standard clustering methods, our approach has the advantages that $i$ ) it is parameter free, ii) the number of clusters need not be fixed in advance and iii) the interpretation of the results is transparent. In order to test our approach and compare it with standard clustering algorithms, we analyze two very different data sets: Time series of financial market returns and gene expression data. We find that different maximization algorithms produce similar cluster structures whereas the outcome of standard algorithms has a much wider variability.
\end{abstract}

Keyword Dataclustering; Econophysics; Gene expression PACS 02.50.Le, 05.40.+j, 64.60.Ak, 89.90.+n

\section{Introduction}

One aspect of the Information Technology revolution is that huge amounts of data have become available. For example, every transaction in many financial markets is recorded and bio-informatics technology allows us today to monitor genome wide gene expression. These informations may allow for a quite detailed description of such complex systems as a financial market or a cell, to quote just two examples. However our understanding of a complex systems is, in many cases, limited by our ability to efficiently organize massive streams of information. As a result, methods for handling, organizing or mining large data sets have become of great practical importance.

Data clustering deals with the problem of classifying a set of $N$ objects into groups so that objects within the same group are more similar than objects belonging to different groups. Each object is identified by a number $D$ of measurable features: Hence object $i=1, \ldots, N$ can be represented as a point $\vec{x}_{i}=$ $\left(x_{i}^{(1)}, \ldots, x_{i}^{(D)}\right)$ in a $D$ dimensional space. Data clustering aims at identifying clusters as more densely populated regions in this vector space. More precisely, a configuration of clusters is represented by a set $\mathcal{S}=\left\{s_{1}, \ldots, s_{N}\right\}$ of integer labels, where $s_{i}$ is the cluster to which object $i$ belongs.

We focus on unsupervised approaches, which do not require any further information apart from that contained in the data. These are of great practical importance specially in the presence of huge data sets. 
We assume, in other words, that the data set is homogeneous: all features are equally important or are already appropriately weighted.

The classic approaches to data clustering are partitioning methods and hierarchical clustering. Partitioning methods are based on two elements: 1) a distance between objects, which allows one to measure their similarity and 2) a cost function whose minima correspond to "optimal" clustering configurations. For example, a typical K-means (KM) approach takes as cost function the sum of squared distances of objects to the centroid $\vec{X}_{s}$ of the cluster in which they are classified [1]:

$$
H_{K M}\{\mathcal{S}\}=\sum_{s} \sum_{i: s_{i}=s}\left\|\vec{x}_{i}-\vec{X}_{s}\right\|^{2}, \quad \vec{X}_{s}=\frac{1}{n_{s}} \sum_{i: s_{i}=s} \vec{x}_{i}
$$

where $n_{s}=\sum_{i: s_{i}=s} 1$ is the number of objects in cluster $s$, and $\|\vec{x}\|^{2}=\sum_{j}\left(x^{(j)}\right)^{2}$ defines the Euclidean distance.

Apart from cases where a cost function is naturally suggested by the problem itself the choice of the cost function or of a distance is an element of arbitrariness. A further problem of these approaches is that one needs to predefine the number $K$ of clusters from the beginning. Note, for example that $H_{K M}$ of Eq. (11) attains its minimal value $H_{K M}=0$ when each object is in a different cluster $\left(n_{s}=1\right)$. The number $K$ of clusters should then be fixed a priori or one has to introduce a $K$-dependent term in the cost function. This is a further element of arbitrariness.

The problem of finding the cluster structure which minimizes the cost function may be quite difficult: depending on the form of the cost function and on the data set the "cost landscape" can be either simple, with a single minimum which is easily accessible dynamically, or complex, with many metastable minima. Data clustering methods also differ for the specific algorithms used to reach a (local) minimum.

A second, very popular approach to data clustering - called hierarchical clustering - is based on the definition of a distance between objects and clusters of objects and a very simple algorithm: Given a configuration with $K>1$ clusters, it merges the two closest clusters into a single one. In this way, starting from the configuration with $K=N$ clusters, the algorithm generates a sequence of configurations as $K$ varies from $N$ to 1 . This sequence of configurations and their hierarchic organization, can be represented by a convenient and compact graphical tool called dendrogram 11. The minimal spanning tree algorithm - also called Single Linkage (SL) - the Average Linkage (AL) and the Centroid Linkage (CL) algorithms are examples of hierarchical clustering method: ${ }^{2}$. Rather than a single cluster structure, this algorithm provides a hierarchic sequence of cluster structures. The choice of the best cluster structure is left arbitrary. Applications of this approach to real world data is discussed in Refs. [2, 3].

Many other approaches to data clustering have been proposed: For example Refs. [4, 5] used singular value decomposition to identify clusters. Identifying principal components with cluster structures imposes an orthogonality constraint between clusters which may be unnatural. Expectation Minimization is a further approach [12] where the density of points is modeled as a mixture of Gaussians whose centering and scale parameters are fit by maximum likelihood. Kohonen et al. [6] have proposed an algorithm based on SelfOrganizing Maps (SOM) whereas Blatt et al. proposed the Super-Paramagnetic Clustering (SPC) method [7]. The latter is based on a mapping to an interacting particle system whose "magnetic" properties describe the cluster structure of data and has been applied to a range of problems (see e.g. [8, 9, 10]). These methods rely on ad hoc definitions of a dynamics (SOM) or of the particle-particle interaction (SPC) which are tuned by several parameters or functions.

In the following sections we briefly review yet a different approach to dataclustering that we recently proposed [11], we describe possible algorithms based on it, and we finally compare the outcomes with those of standard data clustering methods, as those described above.

\footnotetext{
${ }^{1}$ The typical example is that of deciding the location of $K$ distribution centers which should serve $N$ cities. It is natural to look for the locations which minimize the sum of distances.

${ }^{2}$ These algorithms differ in the way the distance between clusters is computed: In SL (AL) the distance between clusters $s$ and $r$ is the minimal (average) distance between items in cluster $s$ and items in cluster $r$; in CL the distance $\left\|\vec{X}_{s}-\vec{X}_{r}\right\|$ between centroids is used.
} 


\section{Maximum likelihood data clustering}

We have devised [11] a fully unsupervised, parameter free approach to data clustering which derives from a maximum likelihood (ML) principle. The key idea is that objects are similar if they have something in common. In a correct classification, objects belonging to the same cluster should share a common component:

$$
\vec{x}_{i}=g_{s_{i}} \vec{\eta}_{s_{i}}+\sqrt{1-g_{s_{i}}^{2}} \vec{\epsilon}_{i}
$$

Here $\vec{x}_{i}$ is the vector of features of object $i$, normalized so that $\sum_{t} x_{i}^{(t)}=0$ and $\left\|\vec{x}_{i}\right\|^{2}=\sum_{t}\left[x_{i}^{(t)}\right]^{2}=D$ for all $i=1, \ldots, N$; $s_{i}$ is the label of the cluster to which it belongs. $\vec{\eta}_{s}$ is the vector of features of cluster $s$ and $g_{s}$ tunes the similarity of objects within cluster $s$ : For $g_{s}=1$ all objects with $s_{i}=s$ are identical whereas when $g_{s}$ is small objects are very different. The cluster index $s$ ranges from 1 to $N$ in order to allow also for the case of $N$ clusters of one object each. $\vec{\epsilon}_{i}$ describes the deviation of the features of object $i$ from the cluster's features and measurement errors. We take Eq. (2) as a statistical hypothesis and assume that both $\vec{\eta}_{s}$ and $\vec{\epsilon}_{i}$ (for all $i, s=1, \ldots, N$ ) are Gaussian vectors with zero mean and variance $E\left[\left(\eta_{s}^{(t)}\right)^{2}\right]=E\left[\left(\epsilon_{i}^{(t)}\right)^{2}\right]=1$. For any given set of parameters $(\mathcal{G}, \mathcal{S})=\left(\left\{g_{s}\right\},\left\{s_{i}\right\}\right)$ it is possible 111 to compute the probability (density) $P\left(\left\{\vec{x}_{i}\right\} \mid \mathcal{G}, \mathcal{S}\right)$ of observing the data set $\left\{\vec{x}_{i}\right\}$ as a realization of Eq. (2). From this it is possible [11] to compute the likelihood $P\left(\mathcal{G}, \mathcal{S} \mid\left\{\vec{x}_{i}\right\}\right)$. It turns out 11] that the resulting expression depends only on the Pearson's coefficient

$$
C_{i, j}=\frac{\vec{x}_{i} \cdot \vec{x}_{j}}{\sqrt{\left\|\vec{x}_{i}\right\|^{2}|| \vec{x}_{j} \|^{2}}}
$$

For a given cluster structure $\mathcal{S}$, the likelihood is maximal when the parameters $g_{s}$ take the values

$$
g_{s}^{\star}=\sqrt{\frac{c_{s}-n_{s}}{n_{s}^{2}-n_{s}}}
$$

if $n_{s}>1$ and $g_{s}^{\star}=0$ if $n_{s} \leq 1$. Here $n_{s}$ is the number of objects in cluster $s$ and

$$
c_{s}=\sum_{i=1}^{N} \sum_{j=1}^{N} C_{i, j} \delta_{s_{i}, s} \delta_{s_{j}, s} .
$$

Note indeed that when $c_{s} \approx n_{s}$, as for uncorrelated objects, then $g_{s}^{\star} \approx 0$ whereas if objects are very similar, $c_{s} \approx n_{s}^{2}$ and $g_{s}^{\star} \approx 1$.

The maximum likelihood of structure $\mathcal{S}$ can be written as $P\left(\mathcal{G}^{\star}, \mathcal{S} \mid\left\{\vec{x}_{i}\right\}\right) \propto e^{D \mathcal{L}_{c}(\mathcal{S})}$, where the loglikelihood per feature $\mathcal{L}_{c}$ is given by

$$
\mathcal{L}_{c}(\mathcal{S})=\frac{1}{2} \sum_{s: n_{s}>1}\left[\log \frac{n_{s}}{c_{s}}+\left(n_{s}-1\right) \log \frac{n_{s}^{2}-n_{s}}{n_{s}^{2}-c_{s}}\right]
$$

This depends on the original data through the coefficients $c_{s}$ of Eq. (5). The function $\mathcal{L}_{c}$ provides a likelihood measure for cluster structures. The ML structure is that which maximizes $\mathcal{L}_{c}$.

There are several interesting features of $\mathcal{L}_{c}$ :

- If the objects are unrelated $\left(g_{s}^{\star}=0\right.$ or $\left.c_{s}=n_{s}\right)$ or if they are classified in singleton clusters $\left(n_{s}=1\right.$ for all $s$ ) we find $\mathcal{L}_{c}=0$. Loosely speaking, $\max _{\mathcal{S}} \mathcal{L}_{c}(\mathcal{S})$ measures the amount of structure present in the data-set.

\footnotetext{
${ }^{3}$ Equivalently, one may generalize Eq. (2) by adding a constant term $r_{i} \overrightarrow{1}$ and a scale factor $\sigma_{i}$. The maximum likelihood estimates of these parameters are the mean and the variance. Subtracting the mean and rescaling by the variance, leaves us with the normalized data set. The parameters $r_{i}$ and $\sigma_{i}$ are irrelevant as far as the cluster structure is concerned. The latter indeed only depends on the "internal structure" of correlations.

${ }^{4}$ Note that an assumption on the prior probability $P(\mathcal{G}, \mathcal{S})$ is invoked by Bayes formula in this passage. We take $P(\mathcal{G}, \mathcal{S})=$ const which means that every cluster structure $\mathcal{S}=\left\{s_{i}\right\}$ is a priori equiprobable.
} 
- The maxima of $\mathcal{L}_{c}$ do not necessarily coincide with a single cluster containing all objects - as in the SPC approach of Ref. [7] - nor with the configuration with all objects in different clusters - as for $-H_{K M}$ 円

- $\mathcal{L}_{c}$ does not depend on any parameter.

- The number $K$ of clusters is not fixed a priori. Rather it is predicted.

- The interpretation of the results is transparent in terms of the model (2).

Eq. (2) may not be the most appropriate description for a particular data set. In much the same way, a straight line may not be the best description of a set of points on a plane. Still in this example, least squares provide an unambiguous method to compute the coefficients and a statistical measure of the goodness of fit. The same is true for our method: A sharp maximum of $\mathcal{L}_{c} / N$ indicates a robust cluster structure whereas when $\mathcal{L}_{c} / N$ is small and has several local maxima the cluster structure is not statistically significant.

The statistical hypothesis Eq. (2) is specially helpful for high dimensional data sets $(D \gg 1)$ where geometric intuition becomes problematic. From the point of view of the computational cost, the dimensionality $D$ of the data set enters only in the calculation of the matrix $C_{i, j}$. For small $D$ methods based on geometry and visual inspection may be preferrable.

Our approach has some similarity with Expectation Maximization data clustering [12], which is also based on likelihood maximization. However the statistical hypothesis in Ref. 12] is very different from Eq. (2).

\section{Data clustering algorithms}

The function $\mathcal{L}_{c}(\mathcal{S})$ is a measure of likelihood for cluster structures and it can be used to compare cluster structures produced by standard methods. For example, it allows one to compare classifications with a different number of clusters and hence to select the optimal number of clusters in KM, SL, AL or CL algorithms. We shall compare later the cluster structure found in this way with those found with algorithms based on $\mathcal{L}_{c}$ itself. Most importantly in fact, $\mathcal{L}_{c}$ can be used as the basis for clustering algorithms.

A very powerful method to find ML configurations is simulated annealing (SA) 13] with cost function $-\mathcal{L}_{c}$. This is simply implemented via Metropolis dynamics [14] on the dynamical variables $s_{i}$ with progressively decreasing (fictitious) temperature $T$. This method produces a cluster configuration, which, if the annealing schedule is appropriately chosen, has a good chance of being that of maximum likelihood. The behavior of the system as a function of the fictitious temperature is similar to that described in Ref. [7]. However, the SPC method (in its original formulation [7]) yields a trivial configuration with a single cluster in the limit $T \rightarrow 0$. This is a consequence of the mapping of the data set into a particle system with ferromagnetic interactions, corresponding to positive correlations. This forces one to study intermediate temperatures 6 . The ML configuration found in our method when $T \rightarrow 0$ is already non-trivial. Further insight on the cluster structure can be obtained by studying the system at finite $T$, as shown in Ref. [11. For simplicity however, we will limit the present discussion to the $T \rightarrow 0$ limit.

A second possibility is that of using a deterministic maximization (DM) technique. We discuss below a very simple algorithm: Given a configuration $S$, for all $i=1, \ldots, N$ (in some fixed order) propose all moves $s_{i} \rightarrow s$ and compute the corresponding variation $\Delta_{s_{i} \rightarrow s}$ of $\mathcal{L}_{c}$; Find $s_{i}^{\prime}=\arg \max _{s} \Delta_{s_{i} \rightarrow s}$ and set $s_{i}=s_{i}^{\prime}$. Repeat these steps until no change occurs. This will lead in the end to a local maximum of $\mathcal{L}_{c}$ which we shall call the $\mathcal{S}_{D M}$ configuration.

Finally we propose a merging algorithm $(\mathrm{MR})$ in the spirit of hierarchical clustering based on $\mathcal{L}_{c}$.

0 ) Start from $N$ clusters composed of one object each (e.g. $s_{i}=i \forall i=1, \ldots, N$ ).

1) At each step of the algorithm merge two clusters into a single one in such a way that the cost $\mathcal{L}_{c}$ of the resulting configuration is minimal.

\footnotetext{
${ }^{5}$ Note that Eq. (1) can be written as $H_{K M}=2 \sum_{s: n_{s}>0}\left(n_{s}-c_{s} / n_{s}\right)$.

${ }^{6}$ Modifications to take into account negative correlations are suggested in Ref. [ 10 ].
} 
2) Repeat step 1) $N-1$ times until the configuration with a single cluster is reached.

Let $\mathcal{S}_{M R}$ be the state with maximal $\mathcal{L}_{c}$ found with this algorithm. $\mathcal{S}_{M R}$ is not necessarily a local maximum of $\mathcal{L}_{c}$ with respect to single "spin-flip" moves $s_{i} \rightarrow s_{i}^{\prime}$, while $\mathcal{S}_{S A}$ and $\mathcal{S}_{D M}$ are local maxima. Hence, we expect that $\mathcal{L}_{c}\left(\mathcal{S}_{M R}\right) \leq \mathcal{L}_{c}\left(\mathcal{S}_{D M}\right) \leq \mathcal{L}_{c}\left(\mathcal{S}_{S A}\right)$. On the other hand, the hierarchical clustering algorithm is definitely faster than both of the others: Even if it delivers only an approximation to the maximum likelihood, faster execution can be crucial when dealing with huge data sets.

In addition, the hierarchical clustering technique MR offers a very convenient graphical representation of the cluster structure in terms of dendrograms. Usually dendrograms report the tree structure as a function of a resolution length. As this length increases, clusters are merged one by one until a single cluster remains when the resolution exceeds the maximal distance $\max _{i, j}\left\|\vec{x}_{i}-\vec{x}_{j}\right\|$ between data points. We draw the dendrogram as a function of the log-likelihood per feature $\mathcal{L}_{c}$. At each merging step $s, r \rightarrow q$ let $\ell_{s}, \ell_{r}$ be the contribution of clusters $s$ and $r$ respectively to $\mathcal{L}_{c}$ before merging and $\ell_{q}$ be the corresponding cluster $\log$-likelihood of the merged cluster $q$. In the dendrogram, we merge two points at height $\ell_{s}$ and $\ell_{r}$ into a single point at height $\ell_{q}$. There are three types of branching points: i) if $\ell_{q}>\ell_{s}+\ell_{r}$ the step increases the likelihood of the configuration ii) if $\ell_{q}<\ell_{s}+\ell_{r}$ but $\ell_{q}>\max \left(\ell_{s}, \ell_{r}\right)$ the global likelihood decreases but the cluster likelihood increases. Finally iii) steps with $\ell_{q}<\max \left(\ell_{s}, \ell_{r}\right)$ represent unlikely merging moves.

\section{Applications and discussion}

We discuss the application of the above ideas to two different data sets: The first consists of daily returns of the NYSE from January the 1st 1987 to March the 30th 1999. We consider $N=1000$ of the assets traded most frequently throughout this period [15]. If $p_{i}(t)$ is the opening price of asset $i$ in day $t$, we set

$$
x_{i}^{(t)}=\frac{\log p_{i}(t) / p_{i}(t-1)-r_{i}}{\sigma_{i}}
$$

where $r_{i}$ and $\sigma_{i}$ enforce normalization $\left(\vec{x}_{i} \cdot \overrightarrow{1}=0\right.$ and $\left.\left\|\vec{x}_{i}\right\|^{2}=D\right)$. Actually it is convenient to introduce a further linear transformation in order to eliminate the so called "market mode": $\vec{x}_{i}^{\prime}=\alpha_{i}\left(\vec{x}_{i}-\vec{x}_{\text {avg }}\right)$ where $\vec{x}_{\text {avg }}=\sum_{i} \vec{x}_{i} / N$ so that $\sum_{i} \vec{x}_{i}^{\prime}=0\left(\alpha_{i}\right.$ is a coefficient to restore $\left.\left\|\vec{x}_{i}^{\prime}\right\|^{2}=1\right)$.

Secondly we discuss the gene expression data set measured in [16] and already studied in Refs. [2, [4, 8] with a variety of techniques. The data measure the expression of the 2467 genes of known function of the yeast Saccharomyces cerevisice, whose complete genome has been sequenced. In particular, we focused on the 18 time values coming from the alpha-factor block-and-release experiment in 16]; these correspond to approximately two cell cycles. Also in this case, we take the logarithm of the value of the gene expression, so that the data distribution is closer to a Gaussian, and normalize the sets as required by Eq. 2 .

In both cases the data set is homogeneous by definition: features characterize the system - the market or the cell - at different times and there is no a priori reason to weight features differently? The main objective of data clustering for these data sets is that of identifying groups of assets with a similar market behavior or groups of genes with a similar function. This information is of considerable interest for risk management strategies and for the understanding of biological functions respectively in the two cases. We shall not enter here into details of either finance or genetics, but rather focus on the properties of data clustering algorithms for these two case studies. On one hand we are interested in identifying fast clustering algorithms, on the other it is desirable that the resulting cluster structures does not depend strongly on the detailed algorithm used. In few words, one would like to identify fast algorithms which provide results which are consistent with more elaborate and time consuming ones. Note that the two data sets are very different in nature: For market data time series are very long $(D=3114)$ and correlations are very weak while gene expression is recorded for few $(D=18)$ time points but correlations are very strong. Thus they constitute a good testing ground for our analysis.

The cluster structure which emerges from the minimization of $\mathcal{L}_{c}$ are quite meaningful: For market data we find a strong overlap with the classification of stocks in sectors of economic activity. In the gene expression

\footnotetext{
${ }^{7}$ Note however that some major crashes such as black Monday of October 1987 are included in the financial data set.
} 


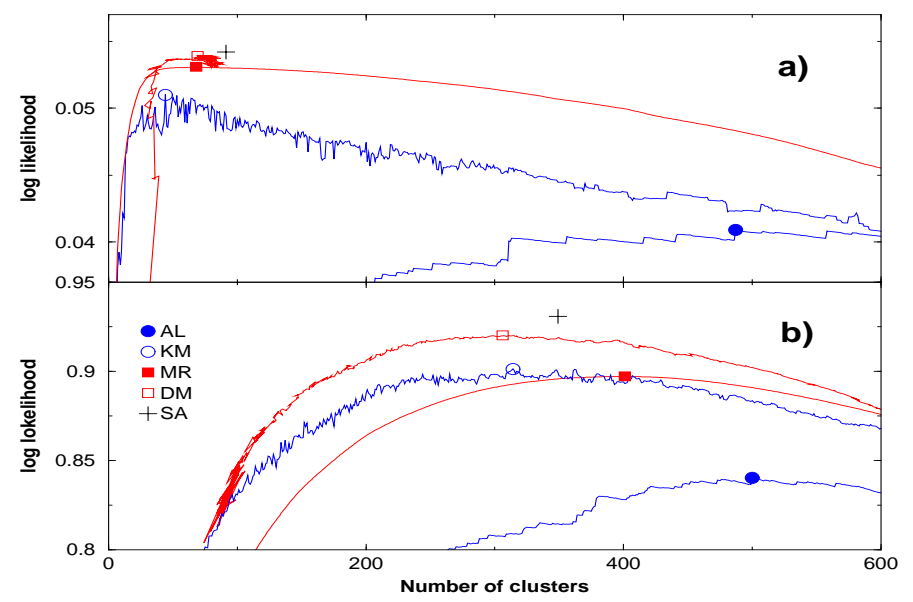

Figure 1: Comparing methods: The log-likelihood $\mathcal{L}_{c}$ is plotted against the number of clusters for the different methods discussed in the text. Points corresponds to the maximal likelihood configurations. The upper plot (a) refers to financial data, the bottom one (b) refers to gene expression data. Configurations $\mathrm{KM}$ and DM are obtained by deterministic minimization of $H_{K M}$ and $-\mathcal{L}_{c}$ respectively, starting from all configurations generated by AL and MR respectively. $K$ is held fixed in the minimization of $H_{K M}$ but not in the maximization of $\mathcal{L}_{c}$. Hence DM can have a number of clusters different from that of the starting MR configuration.

Table 1: Number of clusters $K$, and likelihood per data for the maximum likelihood cluster structures obtained with different methods for the gene expression and the financial datasets.

\begin{tabular}{l|cc|cc}
\multicolumn{2}{c}{ Gene expression } & \multicolumn{2}{c}{ Financial market } \\
Method & $K$ & $\mathcal{L}_{c} / N$ & $K$ & $\mathcal{L}_{c} / N$ \\
\hline Single Linkage (SL) & 1788 & 0.3046 & 885 & 0.0285 \\
Centroid Linkage (CL) & 615 & 0.7806 & 910 & 0.0235 \\
Average Linkage (AL) & 500 & 0.8404 & 487 & 0.0409 \\
K-means (KM) & 314 & 0.9014 & 44 & 0.0510 \\
\hline $\mathcal{L}_{c}$ Merging (MR) & 401 & 0.8972 & 68 & 0.0531 \\
Determ. min $\mathcal{L}_{c}(\mathrm{DM})$ & 306 & 0.9202 & 69 & 0.0539 \\
Sim. Anneal. $\mathcal{L}_{c}(\mathrm{SA})$ & 349 & 0.9308 & 91 & 0.0542 \\
\hline
\end{tabular}

data set, as in Ref. [8], our method identifies clusters related to biological functions. This information is preserved more or less in the ML cluster structures found with different methods.

Standard hierarchical clustering methods (SL, CL and AL) give ML configurations which differ considerably from that found with other methods (see Ref. [15] for details). Table 1 shows that the configurations $\mathcal{S}_{S L}, \mathcal{S}_{A L}$ and $\mathcal{S}_{C L}$ have many more clusters than the others and a much lower value of $\mathcal{L}_{c}$. Fig. 11 compares the log-likelihood of configurations produced in different algorithms as a function of the number of clusters. The curve corresponding to AL deviates markedly from those of other methods; the SL and CL curve (not shown) deviate even more. Not only ML configurations are very different because of a larger number of clusters. Even configurations with a fixed number $K$ of clusters produced by the SL, CL and AL algorithm differ considerably among themselves and from the corresponding configurations with $K$ clusters found with other methods (see Fig. 2).

The closeness of points MR, DM and SA in Fig. 1 1 suggests that methods based on $\mathcal{L}_{c}$ produce consistent results, irrespective of the algorithm. The closeness of two cluster structures $\mathcal{S}$ and $\mathcal{S}^{\prime}$ can be quantified in terms of overlaps: Consider the intersection $\mathcal{S} \cap \mathcal{S}^{\prime}$ of the two cluster structures - defined so that objects $i$ and $j$ are in the same cluster in $\mathcal{S} \cap \mathcal{S}^{\prime}$ only if they are in the the same cluster both in $\mathcal{S}$ and in $\mathcal{S}^{\prime}$. We 


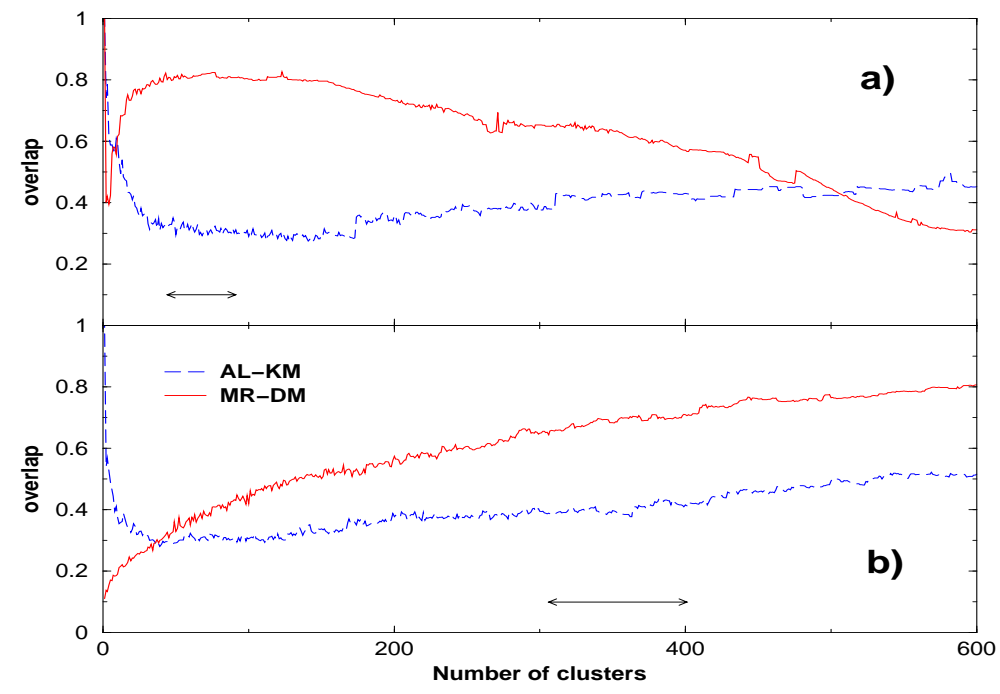

Figure 2: Comparing hierarchical clustering and deterministic minimization algorithms: From the configuration AL (MR) with $K$ clusters found by hierarchical clustering we run deterministic minimization of $H_{K M}$ $\left(\right.$ respectively $-\mathcal{L}_{c}$ ) and find the configuration KM $(\mathrm{DM})$. Overlaps are computed as $\sqrt{O(X \mid Y) O(Y \mid X)}$ (see caption of Table 2) where $(\mathrm{X}=\mathrm{AL}, \mathrm{Y}=\mathrm{KM})$ or $(\mathrm{X}=\mathrm{MR}, \mathrm{Y}=\mathrm{DM})$. The upper plot (a) refers to financial data whereas the bottom one (b) refers to gene expression data. Note that KM has the same number of clusters $K$ as AL but $K$ can be different for DM and MR because $K$ need not be fixed in the maximization of $\mathcal{L}_{c}$. The arrows in the plot indicate the range of $K$ for ML configurations.

Table 2: Geometric overlaps $O_{g}\left(\mathcal{S} \mid \mathcal{S}^{\prime}\right)$ between cluster structures for financial data (with $\mathcal{S}$, $\mathcal{S}^{\prime}$ being the ML cluster structures found with the algorithms SL, AL, KM, MR, DM or SA).

\begin{tabular}{l|ccc|ccc|}
$\mathcal{S}^{\prime} \backslash \mathcal{S}^{\prime}$ & $\mathrm{SL}$ & $\mathrm{AL}$ & $\mathrm{KM}$ & $\mathrm{MR}$ & $\mathrm{DM}$ & $\mathrm{SA}$ \\
\hline $\mathrm{SL}$ & 1 & 0.684 & 0.059 & 0.016 & 0.019 & 0.013 \\
$\mathrm{AL}$ & 0.736 & 1 & 0.220 & 0.081 & 0.088 & 0.081 \\
$\mathrm{KM}$ & 0.938 & 0.465 & 1 & 0.176 & 0.228 & 0.215 \\
\hline $\mathrm{MR}$ & 0.828 & 0.564 & 0.575 & 1 & 0.850 & 0.824 \\
$\mathrm{DM}$ & 0.803 & 0.497 & 0.613 & 0.697 & 1 & 0.916 \\
$\mathrm{SA}$ & 0.532 & 0.440 & 0.555 & 0.649 & 0.882 & 1 \\
\hline
\end{tabular}

define a geometric overlap

$$
O_{g}\left(\mathcal{S} \mid \mathcal{S}^{\prime}\right)=\frac{\left|\mathcal{S} \cap \mathcal{S}^{\prime}\right|}{\left|\mathcal{S}^{\prime}\right|}
$$

where $|\mathcal{S}|$ is the number of pairs of objects that are in the same cluster in configuration $\mathcal{S}$. In words, $O_{g}\left(\mathcal{S} \mid \mathcal{S}^{\prime}\right)$ is the fraction of pairs of objects that are in the same cluster in $\mathcal{S}^{\prime}$ which also belong to the same cluster in $\mathcal{S}$. Equivalently, it is the probability that that two randomly chosen objects which belong to the same cluster in $\mathcal{S}^{\prime}$ are also in the same cluster in $\mathcal{S}$. Tables 2 and 3 report the geometric overlaps between the ML configurations and confirm the conclusion that methods based on $\mathcal{L}_{c}$ yield consistent results. In fact, the overlaps among configurations $\mathrm{MR}, \mathrm{DM}$ and SA are generally larger than those in the sector $\mathrm{SL}, \mathrm{AL}$, KM. These features manifest strongly in weakly correlated data sets, such as market data, and to a milder extent when correlations are stronger, as in the gene expression data set. Still the distribution of cluster sizes produced by SL and AL algorithms is much more uneven than that found with other methods.

The geometric overlap is dominated by clusters which contribute negligibly to the likelihood and which 
Table 3: Same as table 2 for gene expression data. Note that generally, if $\mathcal{S}$ has more clusters than $\mathcal{S}^{\prime}$, one would expect $O\left(\mathcal{S} \mid \mathcal{S}^{\prime}\right)<O\left(\mathcal{S}^{\prime} \mid \mathcal{S}\right)$, provided that the distribution of cluster size is approximately the same. In this example instead, $O(A L \mid \mathcal{S})>O(\mathcal{S} \mid A L)$, for $\mathcal{S}=\mathrm{KM}, \mathrm{MR}$, DM and SA, even if AL has more clusters than $\mathcal{S}$. This is due to the fact that, at odds with configurations $\mathcal{S}$, AL has a very uneven cluster size distribution with two large clusters $\left(n_{s} \approx 40\right)$. The same happens for SL.

\begin{tabular}{l|ccc|ccc|}
$\mathcal{S}^{\prime} \backslash \mathcal{S}^{\prime}$ & $\mathrm{SL}$ & $\mathrm{AL}$ & $\mathrm{KM}$ & $\mathrm{MR}$ & $\mathrm{DM}$ & $\mathrm{SA}$ \\
\hline $\mathrm{SL}$ & 1 & 0.311 & 0.224 & 0.200 & 0.179 & 0.181 \\
$\mathrm{AL}$ & 0.204 & 1 & 0.485 & 0.561 & 0.457 & 0.472 \\
$\mathrm{KM}$ & 0.118 & 0.390 & 1 & 0.433 & 0.454 & 0.463 \\
\hline $\mathrm{MR}$ & 0.075 & 0.322 & 0.310 & 1 & 0.531 & 0.445 \\
$\mathrm{DM}$ & 0.086 & 0.335 & 0.416 & 0.678 & 1 & 0.558 \\
$\mathrm{SA}$ & 0.076 & 0.302 & 0.369 & 0.495 & 0.487 & 1 \\
\hline
\end{tabular}

Table 4: Likelihood overlaps $O_{l}\left(\mathcal{S}^{\prime} \mid \mathcal{S}\right)$ for financial data. Values $O_{l}\left(\mathcal{S}^{\prime} \mid \mathcal{S}\right)>1$ imply that the intersection of the two configuration is more likely than the configuration $\mathcal{S}$.

\begin{tabular}{l|cccccc}
$\mathcal{S} \backslash \mathcal{S}^{\prime}$ & $\mathrm{SL}$ & $\mathrm{AL}$ & $\mathrm{KM}$ & $\mathrm{MR}$ & $\mathrm{DM}$ & $\mathrm{SA}$ \\
\hline $\mathrm{SL}$ & 1.000 & 1.018 & 1.006 & 0.996 & 0.990 & 0.982 \\
$\mathrm{AL}$ & 0.643 & 1.000 & 1.057 & 1.076 & 1.084 & 1.096 \\
$\mathrm{KM}$ & 0.509 & 0.846 & 1.000 & 0.961 & 0.967 & 0.972 \\
$\mathrm{MR}$ & 0.485 & 0.828 & 0.924 & 1.000 & 0.974 & 0.975 \\
$\mathrm{DM}$ & 0.474 & 0.821 & 0.915 & 0.958 & 1.000 & 0.984 \\
$\mathrm{SA}$ & 0.468 & 0.826 & 0.915 & 0.955 & 0.980 & 1.000
\end{tabular}

are therefore not statistically significant. It is then preferable to introduce a likelihood overlap

$$
O_{l}\left(\mathcal{S}^{\prime} \mid \mathcal{S}\right)=\frac{\mathcal{L}_{c}\left(\mathcal{S} \cap \mathcal{S}^{\prime}\right)}{\mathcal{L}_{c}(\mathcal{S})}
$$

which yields the fraction of likelihood of configuration $\mathcal{S}$ which is also "explained" by configuration $\mathcal{S}^{\prime}$. Tables 国 and 5 show that most of the relevant information (in terms of likelihood) gained by standard methods is also contained in ML configurations found with our method. The opposite is however not true.

The discrepancy between results of deterministic minimization and hierarchical clustering algorithms is much reduced if one uses $\mathcal{L}_{c}$. Indeed, MR and DM predict a number of clusters $K$ of the same order whereas AL predicts a much larger $K$ than KM, specially if correlations are weak, as in the financial data set (see table (1). As a further evidence, Fig. 2 compares the overlap between configurations AL and KM with $K$ clusters with the overlap between the configuration MR with $K$ clusters and the corresponding configuration DM (see caption): Configurations MR and DM are more similar than configurations AL and KM.

At odds with SL, AL and CL algorithms, hierarchical clustering yields a ML configurations (MR) which is quite consistent with maximization algorithms (SA or DM) which are computationally more demanding. This, we believe, is due to the fact that $\mathcal{L}_{c}$ generates in typical cases a configuration landscape which is smoother and with more easily accessible maxima, than that generated by conventional distance-based cost functions, such as $H_{K M}(\mathcal{S})$. This conjecture is partially confirmed by looking at simple cases. Take, for example, a data set generated by Eq. (2) with constant correlation $\vec{x}_{i} \vec{x}_{j}=g>0$ for all $i \neq j$. All configurations with two clusters have the same cost $H_{K M}$, and there are $2^{N-1}-1$ such configurations. But the configurations of maximal $\mathcal{L}_{c}$ with two clusters are only those with an isolated object and a large cluster of size $N-1$ (there are $N$ such configurations). Thus $\mathcal{L}_{c}$ gives a much smaller degeneracy than $H_{K M}$ in this case.

The MR algorithm delivers information on the hierarchical organization of the data set. The dendrograms for the two case studies are shown in Figs. 3 and 4 . The dendrogram of market data reveals a complex structure consistent with the scaling laws reported in Ref. [11] (see inset). A much more uniform cluster distribution is found in gene expression data. Figs. 3 and 4 only report the first two types of branch points: 


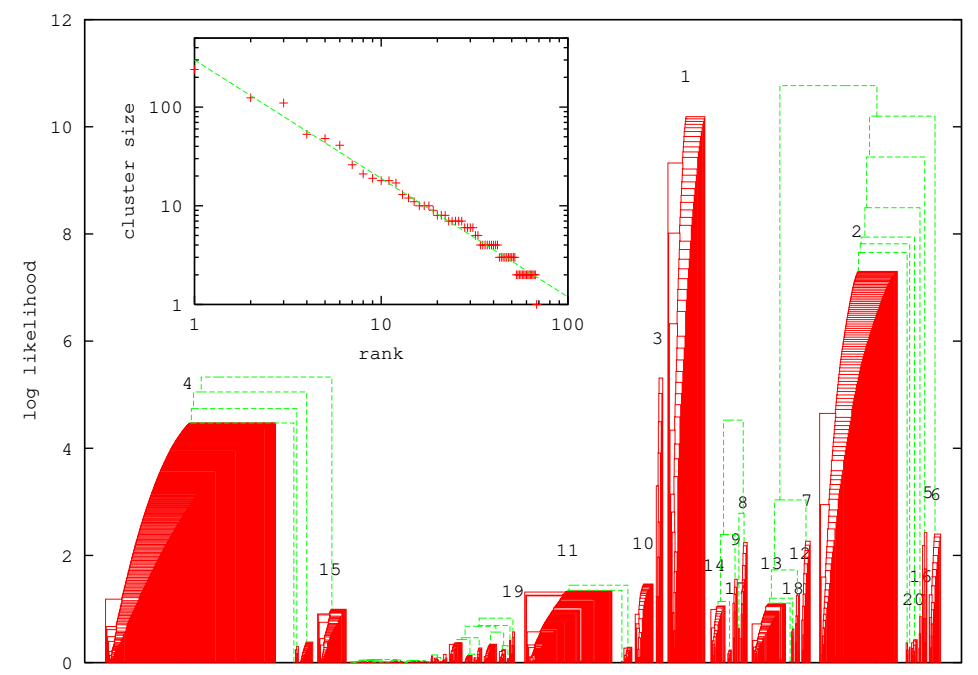

Figure 3: Dendrogram for financial data. The labels report the cluster numbers: For example, cluster 1 contains firms in the electric sector, cluster 3 is the sector of gold, 5 contains telecommunication firms, 6 is composed of banks, 8 and 14 petroleum, 9 oil and gas, 10 computers. Cluster 15 also lists firms in the energy sector. Clusters 2 and 4 are large mixed clusters. Inset: Rank plot of cluster sizes for $\mathcal{S}_{M R}$. The dashed line (drawn as a guide to the eyes) has slope -1.2 which implies that the number of clusters with size larger than $n$ is proportional to $n^{-0.83}$. The same plot for an data set of $N=1000$ uncorrelated time series yields structures with a log-likelihood of $\leq 0.05$. The structure revealed in this plot is on a scale of log-likelihood two orders of magnitude larger.

Table 5: Same as table 4 for gene expression data.

\begin{tabular}{l|cccccc}
$\mathcal{S} \backslash \mathcal{S}^{\prime}$ & $\mathrm{SL}$ & $\mathrm{AL}$ & $\mathrm{KM}$ & $\mathrm{MR}$ & $\mathrm{DM}$ & $\mathrm{SA}$ \\
\hline $\mathrm{SL}$ & 1.000 & 1.192 & 1.078 & 1.201 & 1.158 & 1.149 \\
$\mathrm{AL}$ & 0.432 & 1.000 & 0.808 & 0.925 & 0.862 & 0.843 \\
$\mathrm{KM}$ & 0.364 & 0.753 & 1.000 & 0.736 & 0.782 & 0.761 \\
$\mathrm{MR}$ & 0.408 & 0.866 & 0.739 & 1.000 & 0.851 & 0.792 \\
$\mathrm{DM}$ & 0.383 & 0.788 & 0.766 & 0.830 & 1.000 & 0.810 \\
$\mathrm{SA}$ & 0.376 & 0.761 & 0.737 & 0.763 & 0.801 & 1.000
\end{tabular}

i) those which increase the total likelihood and ii) those which do not increase the total likelihood but where the likelihood of the unified cluster is larger than those of both individual clusters. These two sets of branching points are clearly separate and describe the cluster structure at different resolutions. In most branching of type $i$ ) a large cluster merges with a single object whereas type ii) steps merge clusters into larger clusters.

Gene expression data shows a rich hierarchical structure of type ii) branchings. The ML structure has a large number of clusters with $8 \div 15$ elements. This suggests that the model Eq. (2) does not provide an exhaustive description of the correlations of this data set. There are indeed sizeable correlations between clusters that are not taken into account. An alternative way to describe these correlations, besides that provided by the MR algorithm, is to perform a reclustering of the cluster patterns $\vec{X}_{s}$ with the SA algorithm. More precisely this amounts to taking $\vec{x}_{s}=\vec{X}_{s} /\left\|\vec{X}_{s}\right\|$ as the input data set. Reclustering steps can be iterated until a configuration with singleton clusters is found. This leaves us with a sequence of nested reclustered configurations which provide a hierarchical description of the correlations present in the original data set. In fig. . biological data set. 


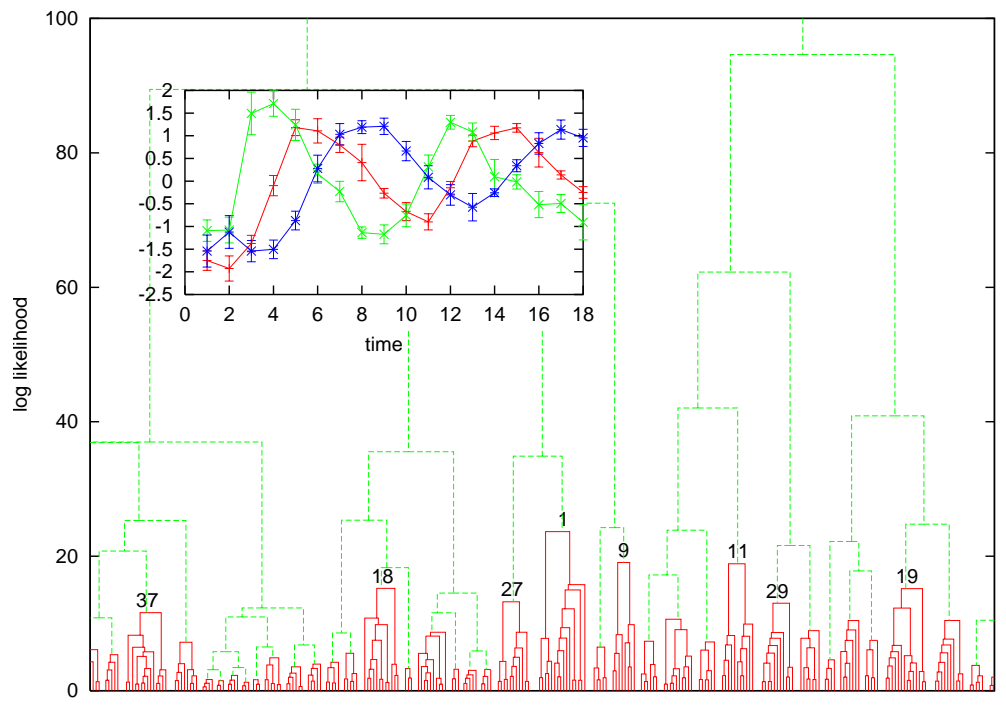

Figure 4: A section of the dendrogram for gene expression data. The labels report the cluster numbers; smaller number indicate higher likelihood. Inset: average patterns of $(\log )$ expression in the first three clusters. Error bars represent the variance inside a cluster. Observe how the clusters identify different patterns with small dispersion. Note that the scale of log-likelihood is an order of magnitude larger than that in Fig. 3 (i.e. 3 orders of magnitude larger than the noise background).

\section{Conclusions}

We have discussed a maximum likelihood approach to data clustering and its application to two examples. The method is based on a simple statistical description of data where similar objects have something in common (Eq. 2). The likelihood that a particular data set is described by such a model with a given cluster structure can be efficiently computed. This provides a parameter-free measure for cluster structures which can then be taken as the basis of clustering algorithms.

Different algorithms to find maxima of the likelihood - or good approximations to them - have been introduced. On one hand we discuss computationally expensive methods, such as simulated annealing technique (SA) and deterministic minimization (DM) algorithms, which are expected to provide a good approximation to the maximum likelihood structure. At odd with most other methods, the number of clusters should not be fixed a priori but is predicted by our algorithms. On the other hand, we introduce a simple deterministic hierarchical clustering algorithm (MR) which provides a much faster approximation.

We show that standard methods with distance based measures of clustering predict cluster structures which may be very different according to the specific algorithm used (SL, AL or KM). On the contrary, the maximum likelihood structures predicted by different algorithms based on the measure $\mathcal{L}_{c}(\mathrm{MR}, \mathrm{DM}$ and SA) are very similar. This suggests that, for instances where a meaningful cluster structure exists, the log-likelihood landscape has a broad and easily accessible maximum, whereas distance-based cost functions such as $H_{K M}$ (Eq. 11) may give rise to a more complex landscape.

Our approach is particularly suited for large dimensional data sets. Indeed, the dimensionality $D$ only enters in the calculation of Pearson's coefficients $C_{i, j}$.

Data clustering has been regarded as an ill defined problem (see e.g. [17]). Indeed in conventional approaches one needs to define a similarity measure and a resolution scale. The approach proposed in the present work does not suffer from these drawbacks. The cluster structure is entirely determined by the internal correlations of the data set. This makes data clustering a well defined problem.

We are grateful to R. N. Mantegna for providing the financial data set. 

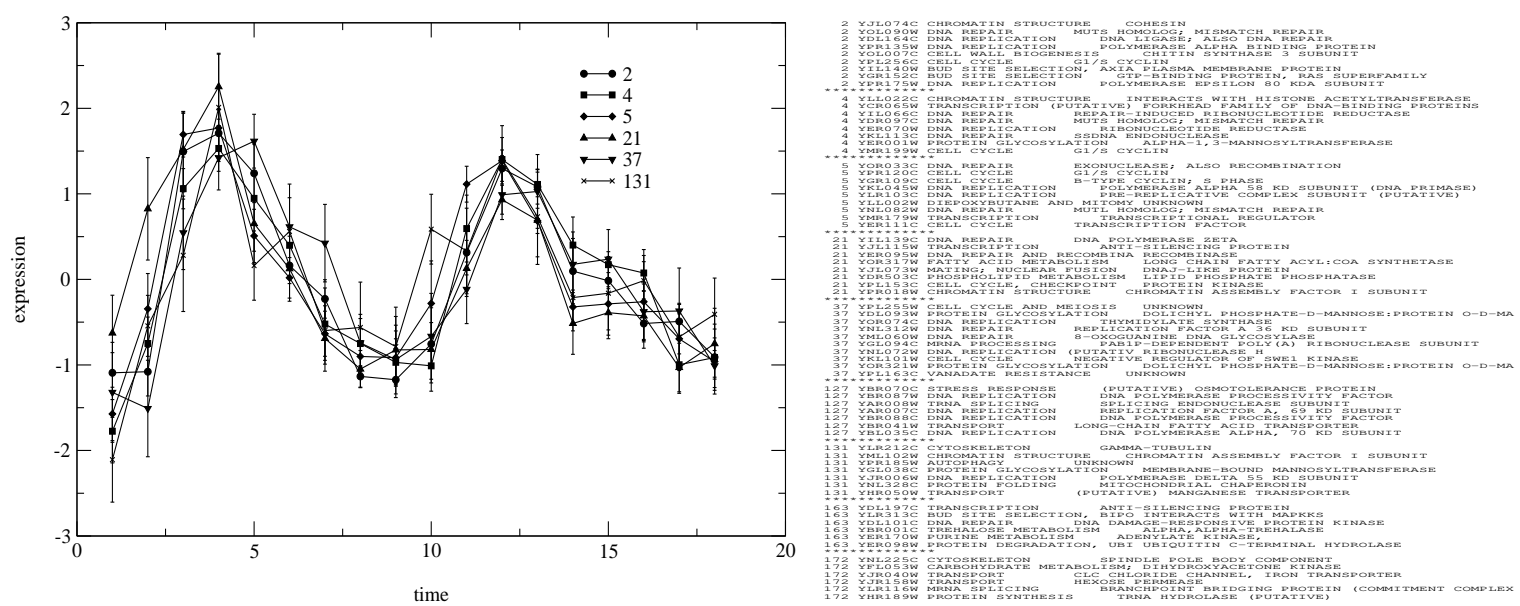

Figure 5: The first 6 elements of cluster number 2 obtained from the second step of reclustering, and the composition of the same cluster. In the table, the first column indicates the number of the cluster after the first step, the second the code of the gene, and the third the corresponding function. At this level of description almost all the information available has been exploited. Observe for example how genes associated to DNA repair, which are put in different clusters after the first step, are found now in the same cluster.

\section{References}

[1] Hartigan, J. (1975) Clustering Algorithms (Wiley, New York).

[2] Eisen, M. B., Spellman, P. T., Brown, P. O. \& Botstein D. (1998) Proc. Natl. Acad. Sci. USA 95, $14863-14868$.

[3] Mantegna, R.N. (1999) Eur. Phys. J. B 11, 193 - 197.

[4] Alter O., Brown P. O., Botstein D. (2000) Proc. Natl. Acad. Sci. USA 97, $10101-6$.

[5] Gopikrishnan, P., Rosenow, B. \& Plerou, V., (2001) Phys. Rev. E 64, 035106

[6] T. Kohonen, K. Mäkisara, O. Simula and J. Kangas (1991), Artificaial Networks, Amsterdam; Tamayo P., Slonim D., Mesirov J., Zhu Q., Kitareewan S., Dmitrovsky E., Lander E.S., Golub T.R. (1999) Proc. Natl. Acad. Sci. USA 96, 2907 - 2912.

[7] Blatt, M., Wiseman, S. \& Domany, E. (1996) Phys. Rev. Lett. 76, 3251 - 3255.

[8] Getz, G., Levine, E., Domany, E. \& Zhang, M. (2000) Physica A 279, 457 - 464.

[9] Getz, G., Levine, E. \& Domany, E. (2000) Proc. Natl. Acad. Sci. USA 97, 12079 - 12084.

[10] Kullmann, L., Kertesz, J. \& Mantegna, R. N. (2000) Physica A 287, 412 - 419.

[11] Giada, L. \& Marsili, M., (2001) Phys. Rev. E 63, 061101.

[12] Lauritzen, S.L. (1995) Computational Statistics and Data Analysis 19, 191 - 201.

[13] Kirkpatrick, S., C. D. Gelatt Jr., M. P. Vecchi, (1983) Science 220, 671 - 680.

[14] Metropolis, N., Rosenbluth. A. W., Rosenbluth, M. N., Teller, A. H. \& Teller, E. (1953) J. Chem. Phys. 21, $1087-1092$. 
[15] Supplementary information, data and downloadable programs can be found on the web page http://www.sissa.it/dataclustering/.

[16] Spellman, P. T., Sherlock, G., Zhang, M. Q., Iyer, V. R., Anders, K., Eisen, M. B., Brown, P. O., Botstein, D. \& Futcher, B. (1998) Mol. Biol. Cell. 9, 3273 - 3297

[17] Domany, E. (1999) Physica A 263,158 - 169. 